\title{
RADIOGRAPHIC AND CLINICAL EVALUATION OF FIXED DETACHABLE PROSTHESES VERSUS TELESCOPIC OVERDENTURES SUPPORTED BY OSSEOINTEGRATED IMPLANTS
}

\author{
Amr Mohamed Ismail Badr*, Ahmed Gamal Ahmed Hassan* \\ and Ahmed Shawkat Rateb Hashem**
}

\begin{abstract}
The purpose of the present study was to evaluate radiographically (the crestal bone level change) and clinically (gingival crevicular fluid and implant stability) in cases with four implants supporting mandibular fixed detachable prostheses versus telescopic overdenture.

Methodology: Twenty completely edentulous patients were treated with four interforaminal implants to support either fixed detachable prostheses with distal cantilevers or telescopic overdenture. The peri-implant marginal bone loss was assessed radiographically at distal surface of posterior implant bilaterally was calculated in two different intervals. The first interval (0-6months) and the second interval (0-12months) using a digital panoramic imaging system. Also clinical evaluation for checking the implant stability using the perio-test and measuring the gingival crevicular fluid.
\end{abstract}

Results: Regarding the gingival crevicular fluid, the comparison between both groups at the time of delivery and after 6 months, there was no significant difference between the two groups. But there was a significant difference after 12 months. From the obtained data of periotest, it was recognized that there was no significant difference between the two groups regarding the implant stability. But there was a significant difference within each group at the different follow-up periods. The data of marginal bone loss at the distal surface of posterior implant on both sides revealed that there was a significant difference between the two groups at the $1^{\text {st }}$ and $2^{\text {nd }}$ intervals.

Conclusion: Both telescopic overdenture and fixed detachable prostheses considered a viable successful treatment option for rehabilitating completely edentulous cases. Telescopic overdentures showed less crestal bone loss at the distal implants than that with the fixed detachable design. The gingival crevicular fluid decreases gradually throughout the study period in both designs denoting successful oral hygiene measurements.

*Lectrurer of Removable Prosthodontics, Faculty of Dentistry, Minia University

**Lecturer of Oral Medicine and Periodontology, Faculty of Dentistry, Damanhour University 


\section{INTRODUCTION}

The state of edentulism can be disabling to the patients and has a negative impact on quality of life..$^{(1,2)}$ Traditionally, the conventional complete denture prostheses were the only solution for restoring edentulous patients. With the innovation of dental implantology, it gives great possibilities in full mouth rehabilitation. ${ }^{(3,4)}$

According to the classic protocol, dental implants must remain covered unexposed to the oral cavity and unloaded during the osseointegration period to prevent the formation of fibrous tissue between bone and implant. ${ }^{(5,6)}$

Telescopic crowns were initially introduced as retainers for removable partial dentures (RPDs) at the beginning of the 20th century. They are also known as a double crown, crown and sleeve coping (CSC), or as Konuskrone, a German term that described a cone shaped design. These crowns consist of an inner or primary telescopic coping, permanently cemented to an abutment or screwed to implants, and a congruent detachable outer or secondary telescopic crown, rigidly connected to a detachable prosthesis. The secondary crown engages the primary coping to form a telescopic unit and serves as an anchor for the remainder of the dentition. ${ }^{(7-9)}$

Implant-supported telescopic overdentures are also considered a viable treatment option for the fully edentulous mandible. Among the indications for an overdenture are deficient retention, unstable prosthesis, poor bone quality, and low economic status of the patient. ${ }^{(10)}$ It also gives an opportunity to reduce destructive rotational and horizontal occlusal forces by directing them more axially.

Overdentures offer many advantages compared with fixed prosthesis and implants, as easy oral hygiene, the possibility of modifying the prosthetic base if necessary, and improved lip support, which enhances esthetics when the maxillary ratio is unfavorable. ${ }^{(11,12)}$
Fixed-detachable hybrid prostheses consist of a metallic framework covered with complete denture components (heat-polymerized resin and denture teeth) that are screwed onto premaxillary or interforaminal implants and consequently incorporate cantilever extensions. ${ }^{(13,14)}$

Although fixed detachable hybrid prostheses offer functional and psychological advantages over removable dentures as they are that they are non removable, the superstructure splints the implants which are thought to distribute occlusal stresses, the tightening screws offer the benefits of providing retention in compromised prosthetic spaces and enabling retrievability. ${ }^{(15)}$ Potential problems such as plaque accumulation, mucositis, peri-implantitis, and/or fracture of acrylic may affect the success rate of these prostheses. Also they may negatively affect the aesthetics, implants' axial load, occlusal stability, and resistance of the veneering material around the screw access holes. ${ }^{(13,16)}$

Rehabilitation of the edentulous jaw with dental implants is a challenge and can improve the physical, psychological, and social well-being of edentulous patients, however consideration must be given to both the type of restoration and the design of the implant. In the lower jaw the patient can be offered a removable overdenture or fixed prosthesis. The type of restoration selected depends on the clinical requirements and economics.

The aim of the present study was to evaluate radiographically (the crestal bone level change) and clinically (gingival crevicular fluid and implant stability) in cases with four implants supporting mandibular fixed detachable prostheses versus telescopic overdenture.

\section{MATERIALS AND METHODS}

Twenty completely edentulous patients were selected from the outpatient clinic, Faculty of Dentistry, Minia University. Eligible patients needed to have bone volumes allowing the placement of 4 
implants with a diameter of at least $3.7 \mathrm{~mm}$ and length of $11.5 \mathrm{~mm}$, between the mental foramina. Patients were not accepted into the study if any of the following exclusion criteria were present: uncontrolled diabetes; pregnancy or lactation; addiction to alcohol or drugs; if they smoked more than 10 cigarettes/day; psychiatric problems; had a severe bruxism habit ; irradiated in the head and/ or neck; treated or under treatment with intravenous amino-bisphosphonates; an active infection or severe inflammation in the area intended for implant placement; need for bone-augmentation procedures or physical handicaps that would interfere with good oral hygiene.

In the present study all patients were treated with four interforaminal implants to support either fixed detachable prostheses with distal cantilevers or telescopic overdenture. Each patient was given a detailed description of the procedures and signed an informed consent document prior to participation in this study.

All patients were operated under local anesthesia according to a strict surgical protocol to receive four root formed tapered threaded dental implants(sclean tapered dental implant fixture-Dentis-Korea) inserted at the mandibular canine and the second premolar area bilaterally.

After reflection of the flap, a surgical stent was applied over the mandibular ridge and used to slightly retract the soft tissue. Great care was taken to maintain parallelism between the implants in bucco-lingual as well as mesio-distal directions using the paralleling tools. The initial osteotomy site was expanded using the successive drills then final preparation was carried out with the final drill at very low speed with copious cooling. The implants were manually inserted using the wrench until the neck was flushed with the crestal bone. External coolant was applied during tightening the implant to avoid overheating the bone.

After a healing period of 3 months, a second surgery to expose the implants was done and impression copings were used for secondary impression by the open tray technique. The verification jig was made intraorally using duralay material. After the impression was poured, the master cast was obtained and verified for accuracy using the verification jig by single screw test.

\section{Prosthetic Procedures}

The patients were randomly divided into two groups, the first group received telescopic overdenture while the second group received fixed detachable prostheses.

\section{Group I (telescopic overdenture):}

The anti-rotational plastic cap (Dentis, antirotational plastic cap, dentis- Korea) attached to each implant analogue. The wax pattern of the primary coping was built up using milling wax (Fraswasles milling wax, BEGO-Germany). The primary copings had parallel side walls and the length and width were adjusted according to each case. A deep chamfer finish line was made at the lower margin of each waxed up coping. The wax pattern was sprued, invested, burnt-out and finally cast into cobalt chromium alloy (cobalt chromium metal framework, vita, Switzerland). The wax pattern of the secondary copings with the metal framework was cast as one piece using the same cobalt-chromium alloy.

Jaw relation, setting up of modified anatomic lower denture teeth and waxing up of the trial denture base was done with the principles of maximum extension. Then flasking, packing, curing, deflasking, finishing and polishing of the lower denture was made. (Fig. (1)(A,B)

The Passive fit between the lower denture with secondary copings and the metallic primary copings was checked. Also, the occlusion was checked by laboratory and clinical remounting. 


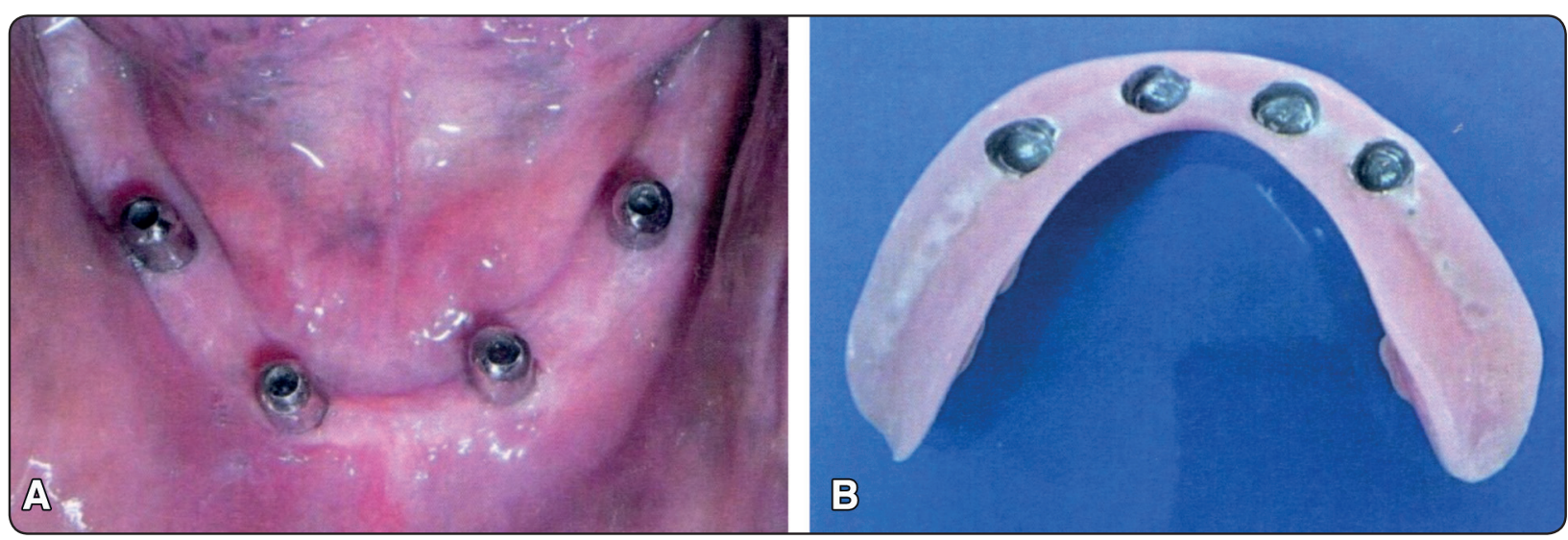

Fig. (1) (A) Intraoral view of telescopic primary abutments. (B) The fitting surface of telescopic overdenture.

\section{Group II (fixed detachable prostheses):}

Patients of this group received four plastic castable abutments screwed to implant analogs splinted with blue inlay wax and cantilevered posteriorly with no more than 1.5 times the antero-posterior distance between the mesial and distal implants. Then cast into cobaltchromium framework. Try in of the metal framework was made then Jaw relation, setting up of modified anatomic lower acrylic teeth and waxing up was done then followed by flasking, packing, curing, deflasking, finishing and polishing of the fixed detachable hybrid prostheses was made. (Fig. (2) A, B, C,D)

The peri-implant marginal bone loss was assessed radiographically at distal surface of posterior implant bilaterally was calculated in two different intervals. The first interval (0-6months) and the second interval (0-12months) using a digital panoramic imaging system (Orthophos XG Plus, Sidexis, Siemens, Sirona Dental Systems). The reduction of the bone height level was determined in relation to the implant shoulder. For this purpose, the initial postoperative radiograph at the loading time (baseline) was compared with the most recent radiograph to calculate peri-implant crestal bone levels and the effective marginal bone loss as the result of the difference. (Fig. 3)

The periotest-M device (Periotest-M, Model 3218, Medizintechnik Gulden e.k. Eschenwig 3,
64397 Modautal, Germany) was used to evaluate the degree of implant mobility. An electrically driven and monitored tapping head percusses the test object 16 times then shows the obtained value. The periotest values are interpreted according to the following: (-8 to 0$)$ good osseointegration, $(+1$ to +9$)$ fair osseointegration, $(+10$ to +50$)$ insufficient osseointegration.

Gingival crevicular fluid (GFC) was collected from buccal surface of the implants at each follow up visit. Whatman filter papers or strips $2 \mathrm{~mm} . \times 1 \mathrm{~cm}$ were used. (Whatman International Ltd Maidstone, England). Each strip was weighted in an empty sterilized plastic Eppendorf tube using an electronic scale before and after collecting the gingival fluid. (Fisher scientific electronic scale).

The implant was isolated with cotton rolls and dried with gentle stream of air for 10 seconds as recommended by Normann ${ }^{(17)}$. The filter paper was introduced into the pocket or sulcus till mild resistance was felt and maintained in place for 3 minutes. Any strip that had been contaminated with blood was discarded as recommended by Nakashina ${ }^{(18)}$. The filter paper with the same plastic Eppendorf tube was reweighted after collection of GCF. The difference between the two weights was considered to equal the volume or weight of the collected fluid as the specific gravity is nearly one; therefore volume $=$ weight. ${ }^{(19)}$ 

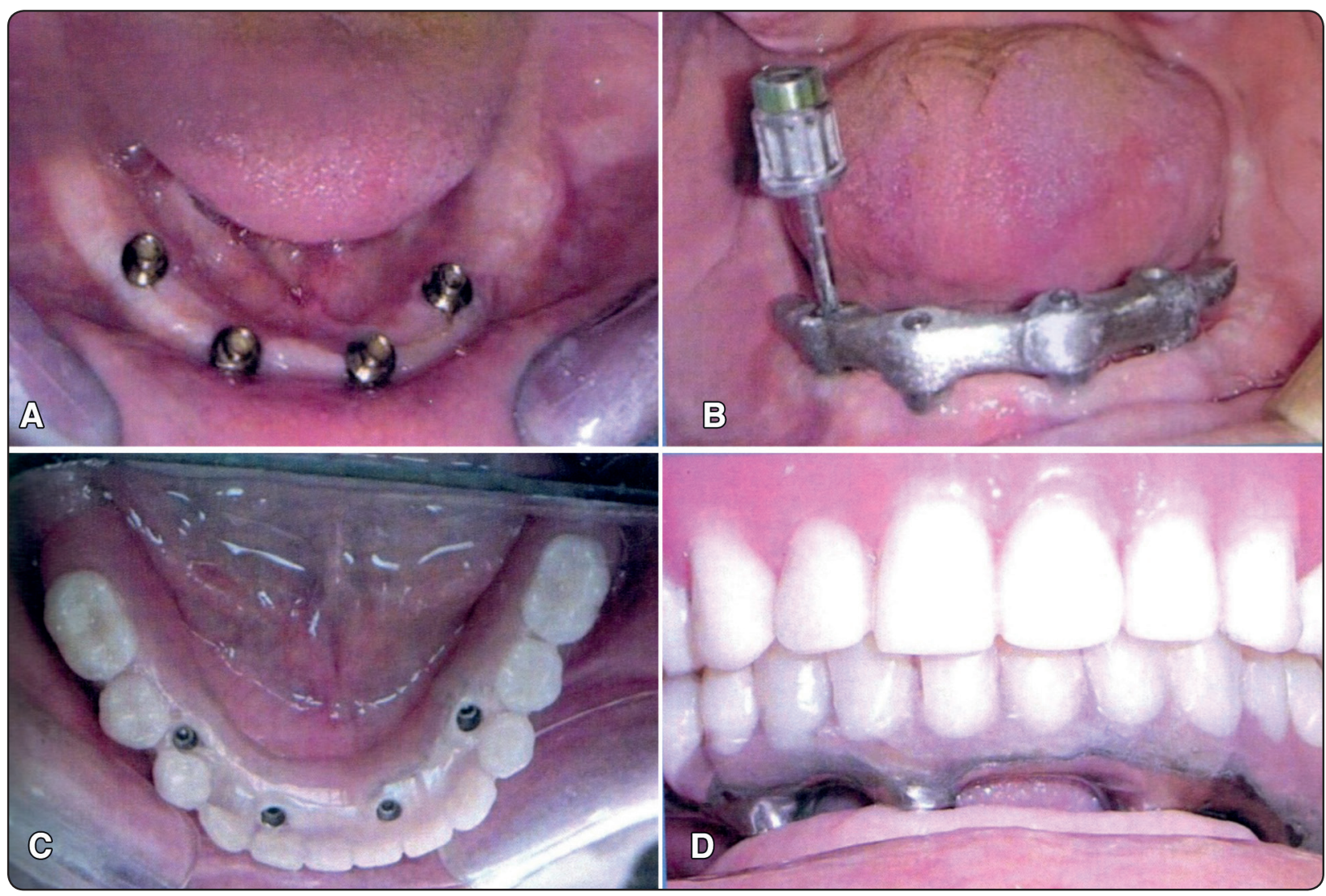

Fig. (2) (A) Intraoral view of abutments receiving fixed detachable prostheses. (B) Intraoral metal framwork try-in using single screw test. (C) Intraoral occlusal view of fixed detachable prostheses screwed to the implants. (D) Intraoral frontal view of fixed detachable prostheses showing the spaces needed for hygienic purpose.

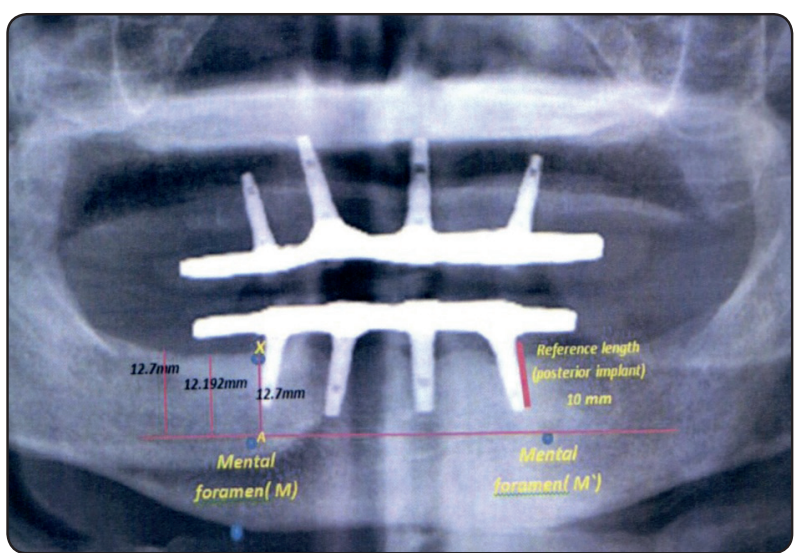

Fig. (3) Panoramic radiograph (1:1) ratio for measuring the crestal bone level.
Statistical analysis was performed using SPSS version 16.0 (SPSS Inc., Chicago, IL, USA). Statistical evaluations: the groups were evaluated using the paired sample $\mathrm{t}$ - test. $\mathrm{P}$ values $<0.05$ indicated statistical significance.

\section{RESULTS}

No implant failure was found during the entire study period. There was no patient who discontinued or dropped out from the study.

\section{Gingival crevicular fluid:}

For the telescopic overdenture group, the data of gingival crevicular fluid revealed that there was no significant difference between the date 
of delivery and after 6 months $(0.017017 \pm 0.002$ and $0.015571 \pm 0.001$ ). But there was a significant difference between the crevicular fluid measurements at 6 months and 12 months $(0.015571 \pm 0.002$ and $0.013386 \pm 0.005$ ).

For fixed detachable group, the data revealed that there was no significant difference between the date of delivery and after 6 months $(0.021529 \pm 0.014$ and $0.015014 \pm 0.005)$. But there was a significant difference between the crevicular fluid measurements at 6 months and 12 months $(0.015014 \pm 0.005$ and $0.0081 \pm 0.011)$.

And regarding the comparison between both groups at the time of delivery and after 6 months, there was no significant difference between the two groups. But there was a significant difference after 12 months between the two groups $(0.013386+0.005$ for telescopic and $0.0081+0.011$ for fixed detachable group). (Table 1), (fig.4).

\section{Implant stability using Periotest device:}

From the obtained data it was recognized that there was no significant difference between the two groups regarding the implant stability. But there was a significant difference within each group at the different follow-up periods as shown in table (2), (fig.5).

\section{Radiographic evaluation of marginal bone loss:}

The data of marginal bone loss at the distal surface of the posterior implant on both sides revealed that there was a significant difference between the two groups at the $1^{\text {st }}$ and $2^{\text {nd }}$ intervals.

Also, there was a significant difference within each group between the $1^{\text {st }}$ interval and the second interval as shown in table (3) (fig.6).

TABLE (1) The gingival crevicular fluid measurements of the telescopic overdenture and screw retained prostheses at different follow-up periods

\begin{tabular}{|c|c|c|c|c|c|}
\hline Group & \multicolumn{2}{|c|}{ Telescopic } & \multicolumn{2}{c|}{ Screw retained } & $\mathrm{p}$-value \\
\hline At delivery & $0.017017 \pm 0.002$ & $p$-value & $0.021529 \pm 0.014$ & $\mathrm{p}$-value & 0.439 \\
\hline 6 months & $0.015571 \pm 0.001$ & 0.241 & $0.015014 \pm 0.005$ & 0.182 & 0.808 \\
\hline 12 months & $0.013386 \pm 0.005$ & 0.009 & $0.0081 \pm 0.011$ & 0.007 & 0.034 \\
\hline
\end{tabular}

*Significant difference between the two groups follow-up period $(P<0.05)$.

TABLE (2) Comparison of the periotest readings between the telescopic overdenture and screw retained prostheses

\begin{tabular}{|c|c|c|c|c|c|}
\hline group & \multicolumn{2}{|c|}{ Telescopic } & \multicolumn{2}{c|}{ Screw retained } & p-value \\
\hline At delivery & $-0.14314 \pm 0.418$ & $\mathrm{p}$-value & $-0.17514 \mathrm{~b} \pm 0.219$ & $\mathrm{p}$-value & 0.246 \\
\hline 6 months & $-2.20114 \pm 0.204$ & 0.000 & $-2.28057 \pm 0.121$ & 0.000 & 0.544 \\
\hline 12 months & $-2.45343 \pm 0.405$ & 0.017 & $-2.82 \pm 0.327$ & 0.000 & 0.054 \\
\hline
\end{tabular}

*Significant difference between the two groups follow-up period $(P<0.05)$. 


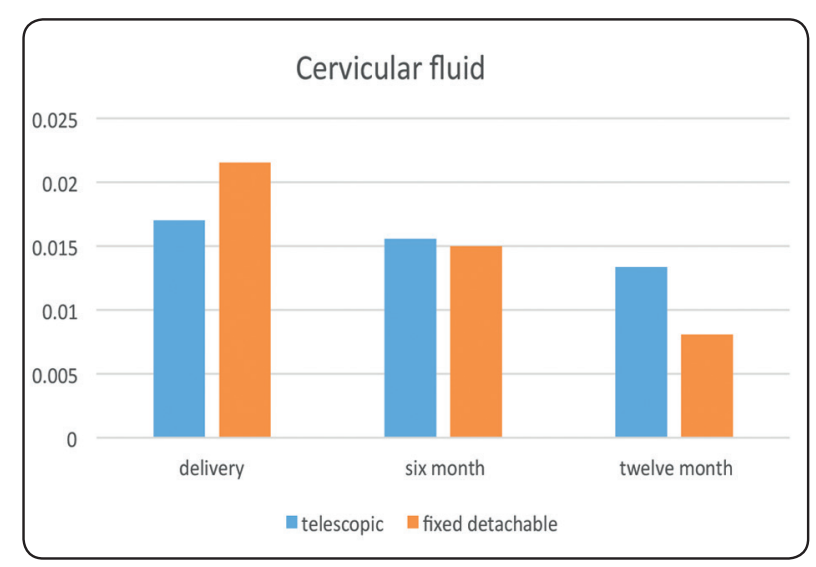

Fig. (4) A bar graph showing the crevicular fluid values of the two groups at different follow-up periods

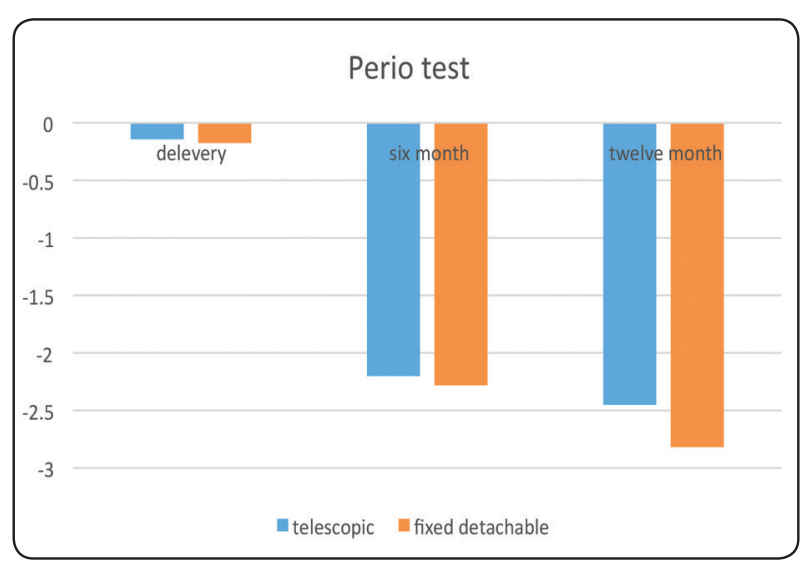

Fig. (5) A bar graph showing the periotest values of the two groups at different follow-up periods

TABLE (3) The marginal bone level change of telescopic overdenture and screw retained prostheses at different follow-up intervals

\begin{tabular}{|c|c|c|c|c|c|}
\hline Group & \multicolumn{2}{|c|}{ Telescopic } & \multicolumn{2}{c|}{ Screw retained } & p-value \\
\hline $1^{\text {st }}$ interval & $0.432429 \pm 0.219$ & $\mathrm{p}$-value & $0.678714 \pm 0.059$ & $\mathrm{p}$-value & 0.011 \\
\hline $2^{\text {nd }}$ interval & $0.688429 \pm 0.121$ & 0.001 & $0.955714 \pm 0.111$ & 0.000 & 0.008 \\
\hline
\end{tabular}

*Significant difference between the two groups follow-up period $(P<0.05)$.

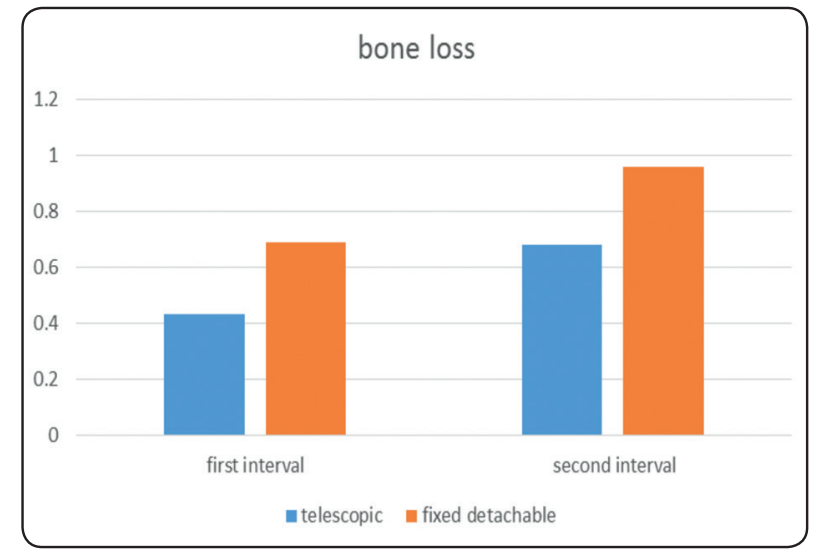

Fig. (6) A bar graph showing the marginal bone level change of telescopic overdenture and screw retained prostheses at different follow-up intervals

\section{DISCUSSION}

All the cases included in this study were successful. No one was dropped out of the study and no failure was recognized.

All the patients subjected to inclusion criteria to nullify all the factors that can affect osseointegration.

Using four interforaminal implants considered an adequate treatment option to restore completely edentulous mandible particularly in cases of posterior ridge resorption.

Initially, the established treatment protocol using fixed screw-retained detachable prostheses for restoring the edentulous mandible primarily relied on the placement of five or six interforaminal implants. (20-22, 25-27) With this treatment protocol, high implant and prosthodontic success rates, as well as strong patient satisfaction scores, 
have been described for cantilevered hybrid detachable screw-retained prostheses. ${ }^{(21-24,28,29)}$ However, in clinical practice, several clinical, technical, and economic considerations have supported a reduction in the number of implants to support fixed restorations. ${ }^{(30-36)}$ With the acceptance of the statement that three or four implants represent the minimum number of implants to be placed for fixed restorations, interest has been focused on mandibular fixed prostheses anchored by a reduced number of implants. ${ }^{(30-32,37,38)}$

Because framework fabrication requires many steps, the clinical and laboratory techniques are complex. The cause of distortion in implant frameworks may be multifactorial. The factors that may introduce errors resulting in distortions include implant alignment, impression technique and materials, framework fabrication process, design configuration, and clinician and technician experience. So the recommended techniques to improve framework fit include the use of custom impression trays, rigid impression materials, one-screw test, and the use of disclosing media. Sometimes to obtain passive fit of the metal substructure may require sectioning and soldering after initial fabrication.

The necessity of abutments parallelism is mandatory in telescopic overdenture for obtaining a common path of insertion and removal.

Marginal bone loss occurs mostly at the distal surface of posterior implant on both sides. That is because this surface is beside the free end saddle area especially when cantilever length is increased.

All the marginal bone loss in both designs was within the acceptable values for implant success. But the results revealed less crestal bone loss at the telescopic groups.

In fixed detachable cases the cantilever arm exerts more torque at the distal surface of posterior implants. Meanwhile, the posterior residual ridge in telescopic overdenture can share the load on the last implant. So this could explain the relatively higher crestal bone loss in fixed detachable design than that of telescopic one.
Ortho-pantographs were taken in this study with 1:1 ratio as it gives a valuable linear measurement.

Radiographic evaluation of crestal bone level around implants by means of a panoramic radiograph may be criticized, because this type of radiograph can be rather imprecise but with recent panoramic machines this concept was changed. This type of radiograph was used routinely in this investigation because intraoral radiographs can be very difficult to obtain in completely edentulous patients, as a result of the very superficial insertion of the muscles of the floor of the mouth and because patients frequently report related discomfort.

Cone beam C.T. was not used in this study as there was no need for measuring the third dimension (bucco-lingual).

The inability to consistently achieve a passive fit with multiple-implant ceramo-metallic prosthesis led to the development of the fixed-detachable hybrid prosthesis. ${ }^{(39,40)}$ As the high temperatures necessary for firing porcelain onto the metal frameworks may result in warpage and lack of passivity. ${ }^{(40,41)}$

A fixed, detachable prosthesis typically is a multiple implant prosthesis that requires a precisely fitting screw-retained framework. An ill-fitting framework will need to be sectioned and soldered or welded to achieve an acceptable fit. ${ }^{(39-42)}$

Also, the hygienic measurements are to some extent difficult for patients receiving fixed detachable prostheses, which adversely affect the amount of gingival crevicular fluid.

At the loading time after the second surgery, the highest crevicular fluid values were seen due to the inflamed mucosal tissues.

The gingival crevicular fluid values starts to decrease gradually from the loading time to 6 months and 12 months respectively.

Regarding the implant stability, the periotest values were acceptable for both designs with no significant difference and continue to improve with time indicating the progression of osseointegration. 


\section{CONCLUSION}

Under the limitation of this study it was concluded that:

1- Both telescopic overdenture and fixed detachable prostheses considered a viable successful treatment option for rehabilitating completely edentulous cases.

2- Telescopic overdentures showed less crestal bone loss at the distal implants than that with the fixed detachable design.

3- The gingival crevicular fluid decreases gradually throughout the study period in both designs

Denoting successful oral hygiene measurements.

\section{REFERENCES}

1. Fiske J, Davis D, Frances C and Gelbier S. The emotional effects of tooth loss in edentulous people. Br Dent J 1998; 184:90-93.

2. Strassburger C, Kerschbaum T and Heydecke G. Influence of implant and conventional prostheses on satisfaction and quality of life: A literature review. Part 2: Qualitative analysis and evaluation of the studies. Int J Prosthodont. 2006; 19:339-348.

3. Turbush S. and Turkyilmaz I. Accuracy of three different types of stereolithographic surgical guide in implant placement: an in vitro study. . J Prosthet Dent. 2012; 3: $181-188$.

4. Ochi M, Kanazawa M, Sato D, Kasugai S, Hirano S and Minakuchi S. Factors affecting accuracy of implant placement with mucosa-supported stereolithographic surgical guides in edentulous mandibles. Computers in Biology and Medicine. 2013; 11: 1653-1660.

5. Brånemark P. Osseointegration and its experimental background. J Prosthet Dent. 1983; 50:399-410.

6. Brånemark P, Hansson B, Adell R, et al. Osseointegrated implants in the treatment of the edentulous jaw. Experience from a 10-year period. Scand J Plast Reconstr Surg Suppl. 1977; 16:1-132.

7. Siegfried M, Alexander S, Friedrich G, Manfred G and HansPeter W. Mandibular two-implant telescopic overdentures-10-year clinical and radiographical results. Clin Oral Implant Res. 2004; 15:560-569
8. Dong $\mathrm{J}$, Ikebe $\mathrm{K}$, Gonda $\mathrm{T}$ and Nokubi T. Influence of abutment height on strain in a mandibular overdenture. J Oral Rehabil .2006; 33:594-599

9. Shah N, Ram S, Mhatre S, and Mahadevan J. Telescopic overdenture with customized double copings-a simplified approach to preventive prosthodontics," Journal of Contemporary Dentistry, vol. 1, pp. 29-32, 2012.

10. Purcell B, McGlumphy E, Holioway J and Beck F. Prosthetic complications in mandibular metal-resin implantfixed complete dental prostheses: a $\mathrm{S}$ to 9-year analysis. Int J Oral Maxillofac Implants. 2008; 23:847-857.

11. Sadowsky S. Mandibular implant-retained overdentures: a literature review. J Prosthet Dent. 2001; 86:468-473.

12. Cune M, de Putter C and Hoogstraten J. Treatment outcome with implant-retained overdentures: part Iclinical findings and predictability of clinical treatment outcome. J Prosthet Dent. 1994; 72:144-151.

13. Misch C. Contemporary Implant Dentistry. 3rd ed. Mosby: St. Louis; 2007.

14. Cobb G, Metcalf A, Parsell D and Reeves G. An alternate treatment method for a fixed-detachable hybrid prosthesis: a clinical report. Journal of Prosthetic Dentistry 2003; 89:239-43.

15. Turkyilmaz I, Company A and McGlumphy E. Should edentulous patients be constrained to removable complete dentures? The use of dental implants to improve the quality of life for edentulous patients. Gerodontology 2010; 27:3-10.

16. Oyagu“ R, Turrio A, Toledano M, Monticelli F and Osorio $\mathrm{R}$. In vitro vertical misfit evaluation of cast frameworks for cement-retained implant-supported partial prostheses. Journal of Dentistry 2009; 37:52-8.

17. Normann H, Staller A, Donna C, Karras and Lonnie K. Reliability of gingival crevicular fluid measurements taken in the presence of supragingival plaque , Journal of periodontology 90:670-673,1990.

18. Nakashina K, Roehrich N and Cimasoni G. Osteocalcin, Prostaglandin E2 and Alkaline Phosphatase in gingival crevicular fluid. Their relation to periodontal status. Journal of clinical Periodontology 21:327-333, 1994.

19. Giannopoulou C, Demenisse C and Cimasoni G. Neutrophils elastase and its inhibitors in human gingival crevicular fluid during experimental gingivitis of dental research 71:359-363, 1992. 
20. Adell R, Lekholm U, Rockler B and Brånemark P. A 15-year study of osseointegrated implants in the treatment of the edentulous jaw. Int J Oral Surg 1981; 10:387-416.

21. Lindquist L, Carlsson G and Jemt T. A prospective 15-year follow-up study of mandibular fixed prostheses supported by osseointegrated implants. Clinical results and marginal bone loss. Clin Oral Implants Res 1996; 7:329-36.

22. Ekelund J, Lindquist L, Carlsson G and Jemt T. Implant treatment in the edentulous mandible: A prospective study on Brånemark system implants over more than 20 years. Int J Prosthodont 2003; 16:602-808.

23. Attard $\mathrm{N}$ and Zarb G. Long-term treatment outcomes in edentulous patients with implant-fixed prostheses: The Toronto study. Int J Prosthodont 2004; 17:417-424.

24. Engfors I, Ortorp A and Jemt T. Fixed implant-supported prostheses in elderly patients: A 5-year retrospective study of 133 edentulous patients older than 79 years. Clin Implant Dent Relat Res 2004; 6:190-198.

25. Collaert B and De Bruyn H. Early loading of four or five Astra Tech fixtures with a fixed cross-arch restoration in the mandible. Clin Implant Dent Relat Res 2002; 4:133-135.

26. De Bruyn H and Collaert B. Early loading of machinedsurface Brånemark implants in completely edentulous mandibles: Healed bone versus fresh extraction sites. Clin Implant Dent Relat Res 2002; 4:136-142.

27. Friberg B and Jemt T. Rehabilitation of edentulous mandibles by means of five TiUnite implants after onestage surgery: A 1-year retrospective study of 90 patients. Clin Implant Dent Relat Res 2008; 10:47-54.

28. Allen P and McMillan A. A review of the functional and psychosocial outcomes of edentulousness treated with complete replacement dentures. J Can Dent Assoc 2003; 69:662.

29. Brennan M, Houston F, O'Sullivan M and O'Connell B. Patient satisfaction and oral health-related quality of life of implant overdentures and fixed complete dentures. Int J Oral Maxillofac Implants 2010; 25: 791-800.

30. Eliasson A, Palmqvist S, Svenson B and Sondell K. Five-year results with fixed complete-arch mandibular prostheses supported by 4 implants. Int J Oral Maxillofac Implants 2000; 15:505-510.

31. De Bruyn H, Kisch J, Collaert B, Lindén U, Nilner K and Dvärsäter L. Fixed mandibular restorations on three earlyloaded regular platform Brånemark implants. Clin Implant Dent Relat Res 2001; 3:176-184.
32. Capelli M,Zuffetti F, Del Fabbro M and Testori T. Immediate rehabilitation of the completely edentulous jaw with fixed prostheses supported by either upright or tilted implants: A multicenter clinical study. Int J Oral Maxillofac Implants 2007; 22:639-644.

33. Francetti L, Agliardi E, Testori T, Romeo D, Taschieri S and Del Fabbro M. Immediate rehabilitation of the mandible with fixed full prosthesis supported by axial and tilted implants: Interim results of a single cohort prospective study. Clin Implant Dent Relat Res 2008; 10:255-263.

34. Gualini F, Gualini G, Cominelli R and Lekholm U. Outcome of Brånemark Novum implant treatment in edentulous mandibles: A retrospective 5-year follow-up study. Clin Implant Dent Relat Res 2009; 14:330-337.

35. Schwarz S, Gabbert O, Hassel A, Schmitter M, Séché C and Rammelsberg P. Early loading of implants with fixed dental prostheses in edentulous mandibles: 4.5-year clinical results from a prospective study. Clin Oral Implants Res 2010; 21:284-289.

36. Agliardi E, Panigatti S, Clerico M, Villa C and Malo P. Immediate rehabilitation of the edentulous jaws with full fixed prostheses supported by four implants: Interim results of a single cohort prospective study. Clin Oral Implants Res 2009; 21:459-465.

37. Engstrand P, Gröndahl K, Ohrnell L, Nilsson P, Nannmark U and Brånemark P. Prospective follow-up study of 95 patients with edentulous mandibles treated according to the Brånemark Novum concept. Clin Implant Dent Relat Res 2003; 5:3-10.

38. Gallucci G, Doughtie C, Hwang J, Fiorellini J and Weber H. Five year results of fixed implant-supported rehabilitations with distal cantilevers for the edentulous mandible. Clin Oral Implants Res 2009; 20:601-607.

39. Drago C, Saldarriaga R, Domagala D, et al: Volumetric determination of the amount of misfit in CAD/CAM and cast implant frameworks: a multicenter laboratory study. Int J Oral Maxillofac Implants 2010; 25:920-929

40. Wee A, Aquilino S and Schneider R. Strategies to achieve fit in implant prosthodontics: a review of the literature. Int J Prosthodont 1999; 12:167-178

41. Winter W, Mohrle S, Holst S, et al: Bone loading caused by different types of misfits of implant-supported fixed dental prostheses: a three dimensional finite element analysis based on experimental results. Int $\mathrm{J}$ Oral Maxillofac Implants 2010; 25:947-952

42. Tiossi R, Falcao-Filho H, Aguiar J, unior F, et al: Modified section method for laser-welding of ill-fitting $\mathrm{CP}$ Ti and $\mathrm{Ni}-\mathrm{Cr}$ one piece cast implant-supported frameworks. J Oral Rehabil 2010; 37:359-363 